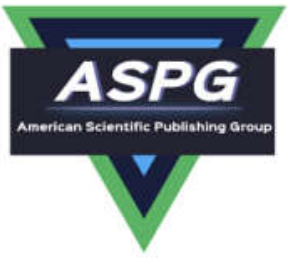

\title{
Indeterminacy in Neutrosophic Theories and their Applications
}

\author{
Florentin Smarandache \\ Mathematics, Physics and Natural Sciences Division, The University of New Mexico, \\ 705 Gurley Ave., Gallup, NM 87301, USA, Email: smarand@unm.edu
}

\begin{abstract}
Indeterminacy makes the main distinction between fuzzy / intuitionistic fuzzy (and other extensions of fuzzy) set / logic vs. neutrosophic set / logic, and between classical probability and neutrosophic probability. Also, between classical statistics vs. neutrosophic and plithogenic statistics, between classical algebraic structures vs. neutrosophic algebrais structures, between crisp numbers vs. neutrosophic numbers. We present a broad definition of indeterminacy, various types of indeterminacies, and many practical applications.
\end{abstract}

Keywords: Indeterminacy, Neutrality, <neutA>, Neutrosophic Triplets, Types of Indeterminacies, Numerical Indeterminacy, Literal Indeterminacy, Neutrosophic Number, Quadruple Neutrosophic Number, Refined Indeterminacy, Subindeterminacies, Null Indeterminacy, Over-/Under-/Off-Indeterminacy, TransIndeterminacies

\section{Introduction}

This paper is written after the author received many questions about the concept of "Indeterminacy" utilized in the neutrosophic theories (such as Neutrosophic Set / Logic / Probability / Statistics / Measure / Precalculus / Calculus / Algebraic Structures), by emails and especially on the very popular websites such as: Researchgate.net, Academia.edu, Facebook, Twitter, and LinkedIn. And after discussions with Dr. Said Broumi and Dr. Nivetha Martin.

The most general definition, the classification, and many real examples of Indeterminacies from our everyday life, utilized in the neutrosophic theories and their applications, are presented below in an understandable manner. "Indeterminacy" should not be taken into the narrow sense of a lexical dictionary, but as something that is in between the opposites.

Because of dealing with various types of indeterminacies (vague, unclear, uncertain, conflicting, incomplete, hesitancy, neutrality, unknown, etc.) related to the data or to the procedures employed in our real world, we may extend by neutrosophication any classical scientific or cultural crisp concept from any field of knowledge to a corresponding neutrosophic (un-crisp) concept, since in our world more things are indeterminate or partially indeterminate than completely determinate.

\section{Neutrosophic Triplets}


Firstly, let's define the neutrosophic triplets.

Let $\langle A>$ be an item (concept, notion, idea, sentence, theory etc.) and $<$ anti $A>$ its opposite. In between the opposites $\langle A\rangle$ and $\langle$ anti $A>$, there is a neutral (or indeterminacy) part, denoted by $<$ neut $A>$.

The $<$ neut $A>$ is neither $<A>$ not $<$ antiA $>$,

or sometimes the $<$ neutA $>$ is a mixture of partial $<A>$ and partial $<$ antiA $>$.

Of course, we consider the neutrosophic triplets $(<\boldsymbol{A}>,<\boldsymbol{n e u t} \boldsymbol{A}\rangle$, $<\boldsymbol{a n t i A}\rangle)$ that make sense in the world, and there are plenty of such triplets in our every day life [1].

\section{Examples of Neutrosophic Triplets}

$>$ (Friend, Neutral, Enemy)

$>$ (Positive, Zero, Negative)

$>$ (Male, Transgender, Female)

$>$ (Win, Tie-game, Lose)

$>$ (Small, Medium, Tall)

$>$ (True, Partially-true \& Partially-false, False)

$>\quad$ (True, Indeterminacy, False)

$>$ (Membership, Partially-membership \& Partially-nonmembership, Nonmembership)

$>$ (White, Red, Black), etc.

\section{Neutrosophic Definition of Indeterminacy}

In neutrosophy, which is a new branch of philosophy, we interpret Indeterminacy in the broadest possible sense, i.e.

Indeterminacy, denoted by $<$ neut $A>$,

is everything that is in between the opposites $\langle A\rangle$ and $\langle$ antiA $\rangle$.

Instead of this general neutrosophic triplet $(\langle A\rangle,\langle$ neut $A>$, $\langle$ antiA $>)$, the neutrosophic community has been mostly using the neutrosophic triplet $(T, I, F)$,

where in a broad sense: $T=$ truth (or membership), $I=$ indeterminacy (unclear, unknown, vague, uncertain, imprecise, etc.), $F=$ falsehood (or nonmembership), with $T, I, F$ as subsets of the interval $[0,1]$.

The word "Indeterminacy" is a generic name for <neutA> (or the letter "I"). It should not be taken literally (in a narrow sense) as in a lexical dictionary (such as Webster, Larousse, etc.).

Indeterminacy depends on each application, or problem to solve, and on the experts. That's why there are many types of Indeterminacies.

In general, Indeterminacy $I$ is not the complement of $T$ and $F$, since the neutrosophic components $T, I, F$ are independent from each other.

As a middle side, $<$ neut $A>$ is neither $<A>$ nor $<$ antiA $>$, but in between them, or sometimes, a combination of them. 


\section{Examples of Indeterminacies}

For the neutrosophic triplet (Friend, Neutral, Enemy), the Indeterminacy = Neutral (i.e. neither Friend nor Enemy).

For the neutrosophic triplet (Positive, Zero, Negative), the Indeterminacy $=$ Zero.

For the neutrosophic triplet (Proton, Neutron, Electron), the Indeterminacy = Neutron.

For the neutrosophic triplet (Positron, Antineutron, Antiproton), the Indeterminacy = Antineutron.

For the neutrosophic triplet (Matter, Unmatter, Antimatter), the Indeterminacy = Unmatter (Unmatter is formed by combinations of matter and antimatter that bound together, or by long-range mixture of matter and antimatter forming a weakly-coupled phase) [12].

For the neutrosophic triplet (Male, Transgender, Female), the Indeterminacy = Transgender (a person whose gender is unclear, indeterminate).

For the neutrosophic triplet (Win, Tie-game, Lose), the Indeterminacy = Tie-game.

For the neutrosophic triplet (Small, Medium, Tall), the Indeterminacy = Medium.

For the neutrosophic triplet (True, Partially-true \& Partially-false, False), the Indeterminacy = Partially-true \& Partially-false (a combination of the opposites).

For the neutrosophic triplet (True, Indeterminacy, False), the Indeterminacy = Indeterminacy.

For the neutrosophic triplet (Membership, Partially-membership \& Partially-nonmembership, Nonmembership),

the Indeterminacy $=$ Partially-membership \& Partially-nonmembership (a combination of the opposites).

For the neutrosophic triplet (Cause, Neither Cause Nor Effect, Effect), the Indeterminacy = Neither Cause Nor Effect.

For the neutrosophic triplet (White, Red, Black), the Indeterminacy $=$ Red.

In Fuzzy Set and Logic, $T=$ the truth (or membership), while $F=1-T=$ the falsehood (or nonmembership), while $I=0$ is the indeterminacy.

In Intuitionistic Fuzzy Set and Logic, $T=$ the truth (or membership), $F=$ the falsehood (or nonmembership), and the indeterminacy is called hesitancy $H=1-T-F$.

In Picture Fuzzy Set and Logic, $T=$ the truth (or membership), $F=$ the falsehood (or nonmembership), and the indeterminacy (I) was split/refined into $N=$ neutrality (or the first subindeterminacy $\mathrm{I}_{1}$ ), and the hesitancy $H=1-T$ $-F-N$ (or the second subindeterminacy $\mathrm{I}_{2}$ ). Therefore: $T, I_{1}=N, I_{2}=H, F$. Picture Fuzzy Set and Logic (also called Inconsistent Intuitionistic Fuzzy Set and Logic, or Ternary Fuzzy Set and Logic)) are particular cases of Refined Neutrosophic Set and respectively Logic (where $T$ is split/refined into $T_{1}, T_{2}, \ldots, T_{p} ; I$ is split/refined into $I_{1}, I_{2}, \ldots, I_{r}$; and $F$ is split/refined into $F_{1}, F_{2}, \ldots, F_{s}$; with integers $p, r, s \geq 0$ and at least one of $p, r$, or $s$ is $\geq 2$; if some $T_{0}, I_{0}, F_{0}$ occur, it is discarded) [3].

Similarly for other fuzzy extension sets and logics \{such as: Pythagorean Fuzzy Set and Logic (also called Atanassov's Intuitionistic Fuzzy Set and Logic of second type), q-Rung Orthopair Fuzzy Set and Logic, Fermatean Fuzzy Set and 
$\underline{\text { Logic, }}$ also Spherical Fuzzy Set and Logic, n-HyperSpherical Fuzzy Set and Logic, etc.\} [13]. They have either two components $(T$ and $F$ ) or three $(T, I$, and $F)$, but with the restrictions that $0 \leq T+F \leq 1$ where what's left $1-T-F$ is indeterminacy, and respectively $0 \leq T+I+F \leq 1$ where what's left $1-T-I-F$ is indeterminacy too.

\section{6. $\underline{\text { Refined Indeterminacy }[3]}$}

In between the opposite $\langle A\rangle=$ White and $<$ anti $A>=$ Black, there is a whole spectrum of colors. In this case, the Indeterminacy $<$ neut $A>$ is split into many Subindeterminacies: $<$ neutAl $>$, $<$ neutA2 $>, \ldots,<$ neutAn $>$, for $n \geq 2$. We have the following $I$-refined neutrosophic triplet (where " $I$-refined" means refinement with respect to Indeterminacy): $(<A>$; $<$ neutAl $>,<$ neutA2 $>, \ldots,<$ neutAn $>$; <antiA $>)$.

Therefore, the (total) Indeterminacy is the union ( $U$ )of all Subindeterminacies:

$$
<\text { neutA }>=<\text { neutA1 }>U<\text { neutA2 }>U \ldots U<\text { neutAn }>\text {. }
$$

\section{Example of Refined Indeterminacy}

For the I-refined neutrosophic triplet (White; Yellow, Pink, Red, Blue, Violet; Black), the Indeterminacy $=$ Yellow U Pink U Red U Blue U Violet.

And the subindeterminacies are: $<$ neutA $1>=$ Yellow, $<$ neutA $2>=$ Pink, $<$ neutA3 $>=$ Red, $<$ neutA4 $>=$ Blue, and $<$ neutA5 $>$ Violet.

There also is possible to have an infinite $I$-refined neutrosophic triplet by considering the infinite color spectrum between White and Black.

\section{The Neutrosophic Logic Triplet [1]}

The Neutrosophic Logic (NL) truth-value of a proposition $P$ is:

$N L(P)=(T, I, F)$, where $T=$ the degree of truth of the proposition $P$;

$$
\begin{aligned}
& I=\text { the indeterminate-degree of the proposition } P \text { to be true or false; } \\
& F=\text { the degree of falsehood of the proposition } P
\end{aligned}
$$

or $T=$ truth, $I=$ indeterminacy, $F=$ falsehood. We prefer to use these descriptive notations $T, I, F$ all over for the neutrosophic components.

\section{The Neutrosophic Set Triplet}

The Neutrosophic Set (NS) membership-value of an element $x$ with respect to a give set $M$ is:

$$
N S(x)=(T, I, F)
$$

where

$T=$ the degree of membership of the element $x$ with respect to the set $M$;

$I=$ the indeterminate-degree of membership or nonmembership of the element $x$ with respect to the set $M$; 


\section{$F=$ the degree of nonmembership of the element $x$ with respect to the set $M$;}

or $T=$ membership, $I=$ indeterminacy, $F=$ nonmembership.

\section{The Neutrosophic Probability Triplet [4]}

The Neutrosophic Probability (NP) of an event $A$ to occur is:

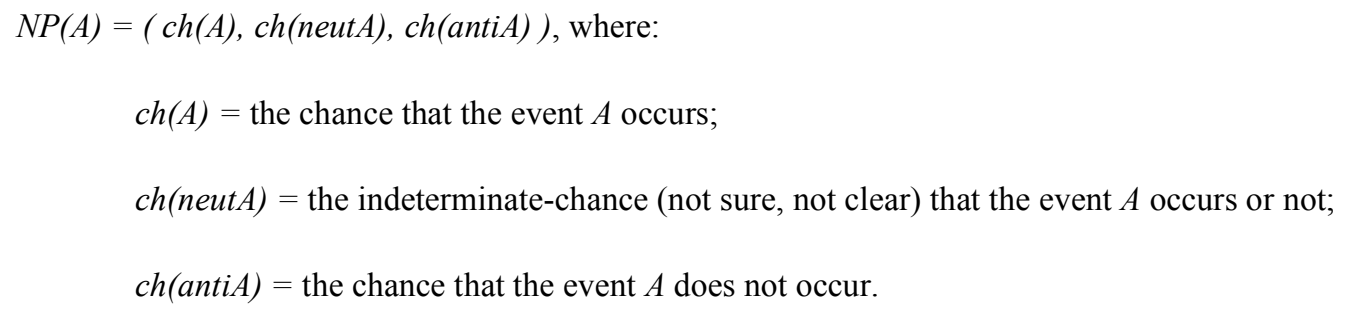

In this case, the Indeterminacy $=\operatorname{ch}($ neut $A)$.

\section{Indeterminacy in Neutrosophic Statistics [5, 6]}

While the Classical Statistics deals with determinate data, determinate probability distributions, and determinate inference methods only, the Neutrosophic Statistics may deals with indeterminate data \{i.e. data that has some degree of indeterminacy (unclear, vague, partially unknown, contradictory, incomplete, etc.) $\}$, indeterminate probability distributions, and indeterminate inference methods \{i.e. distributions and inferences that contain some degrees of indeterminacy as well (for example, instead of crisp arguments and values for the probability distributions and inference methods, charts, diagrams, algorithms, functions etc. one may deal with inexact or ambiguous arguments and values) $\}$.

For example:

- $\quad$ The sample's size or population's size are not exactly known (for example, the size may be between $200-$ 250 individuals).

- Not all individuals may belong $100 \%$ to the sample or populations, some may only partially belong (their degree of belongingness $T<1$ ), others may over-belonging (their degree of belongingness $T>1$ ).

An application:

Upon their work for a factory, John belongs $100 \%$, George 50\%, and Mary $110 \%$ (because she works overtime). John is 40 years old, George 60, and Mary 20. What is the age average of this company's workers?

In the classical statistics, where the degree of belongness to the factory does not count, the age average is simply: $(40+60+20) / 3=40$.

In neutrosophic statistics, where the degree of belongness does count, one has:

$(40 \times 1+60 \times 0.5+20 \times 1.1) /(1+0.5+1.1)=92 / 2.6 \approx 35.38$.

In classical statistics, the degree of belongness was considered $\mathrm{T}=1$ for all workers: but the age average $(40 \times 1+60 \times 1+20 \times 1) /(1+1+1)=120 / 3=40$ is inaccurate, since George's work of only $50 \%$ cannot be the same as Mary's of $110 \%$.

- The distribution probability curves may not be crisp or exactly known (as in classical statistics), but indeterminate functions (with approximations, or vague and conflicting information), or they may be represented by thick functions (the area between two curves).

\section{2. $\underline{\text { When Indeterminacy }=0}$}


Let $T, I, F$ belonging to the interval $[0,1]$ be the neutrosophic components.

If Indeterminacy $I=0$, the neutrosophic components $(T, 0, F)$ are still more flexible and more general than fuzzy components and intuitionistic fuzzy components. Because, we get:

- for the fuzzy set and the intuitionistic fuzzy set (they coincide):

$T+F=1$.

- while for the neutrosophic set:

whence we may have any of these situations:

$$
0 \leq T+F \leq 2
$$

$T+F<2$ (for incomplete information);

$T+F=2$ (for complete information);

$T+F>2$ (for paraconsistent / conflicting information, coming from independent sources).

Therefore, the neutrosophic set is more flexible and more general than the other sets, no matter the value of indeterminacy.

\section{Classification of Indeterminacies}

Since there are many types of indeterminacies, it is possible to define many types of neutrosophic measures in any field of knowledge.

And, in general, because of dealing with lots of types of indeterminacies, we can extend any classical scientific or cultural concept from various indeterminate/neutrosophic viewpoints.

(i) There is the Numerical Indeterminacy, as part of the numerical neutrosophic triplet $(T, I, F)$, when " $I$ " is a numerical subset (interval, hesitant subset, single-valued number, etc.) of $[0,1]$, and it is used in neutrosophic set, neutrosophic logic, and neutrosophic probability.

(ii) And the Literal Indeterminacy, where $I^{\wedge} 2=I$, with " $I$ " just a letter [7], used in neutrosophic algebraic structures (such as: neutrosophic group, neutrosophic ring, neutrosophic vector space, etc.) that are built on the sets of the form:

$S=\left\{a+b I\right.$, with $I^{\wedge} 2=I$, and $a, b$ in $\left.M\right\}$, where $M$ is a given real or complex set.

The Literal Indeterminacy $(I)$ is also used in neutrosophic calculus and in some neutrosophic graphs and neutrosophic cognitive maps, when the edge between two vertexes is unknown and it is denoted by a dotted line (meaning indeterminate edge).

(iii) TransIndeterminacies, inspired from the transreal numbers [11], some of which are:

(a) Infinite Indeterminacy ( denoted by $\infty_{I}$ )

$$
\begin{aligned}
& I^{\infty}=\lim _{n \rightarrow \infty} I^{n}=\infty_{I} \\
& \frac{I}{0}=\lim _{x \rightarrow 0} \frac{I}{x}=\infty_{I} \\
& I \cdot \infty=\infty \cdot I=\lim _{x \rightarrow \infty}(n \cdot I)=\infty_{I}
\end{aligned}
$$

(b) Null Indeterminacy ( denoted by $\phi_{I}$ )

$$
\begin{aligned}
& \frac{I}{\infty}=\lim _{x \rightarrow \infty} \frac{I}{x}=\phi_{I} \\
& I^{-\infty}=\frac{1}{I^{\infty}}=\lim _{n \rightarrow \infty} \frac{1}{I^{n}}=\frac{1}{\infty_{I}}=\phi_{I}
\end{aligned}
$$


(iv) Also, the Neutrosophic Number, $N=d+e \square I$, where $a$ and $b$ are real or complex numbers introduced in [7], and they were interpreted as $N=d+e \square I$, where $d$ is the determinate part of the number $N$, and $e \square I$ is the indeterminate part of the number $N$ in [5].

There are transcendental, irrational etc. numbers that are not well known, they are only partially known and partially unknown, and they have infinitely many decimals. Not even the most modern supercomputers can compute more than a few thousands decimals, but the infinitely many left decimals still remain unknown. Therefore, such numbers are very little known (because only a finite number of decimals are known), and infinitely unknown (because an infinite number of decimals are unknown).

Let's take $\sqrt{3}=1.7320508 \ldots$, then an easy example of neutrosophic number capturing $\sqrt{ }(3)$ is:

$N=a+b \cdot I=1.732+4 \cdot[0.000010,0.000015]=[1.73204,1.73206]$, where of course $a=1.732, b=4$, and $I=$ [0.000010,0.000015].

The way of choosing the parameters $a, b, I$ depends on the needed accuracy of the neutrosophic number $N$, on the problem to solve, and on the experts.

The neutrosophic number is used in neutrosophic statistics, and in neutrosophic precalculus [8].

(v) In the Quadruple Neutrosophic Number, which has the form $Q N=a+b \cdot T+c \cdot I+d \cdot F$, where the known part of $Q N$ is $a$,

and the unknown part of $Q N$ is $b \cdot T+c \cdot I+d \cdot F$,

then the unknown part is split into three subparts:

degree of confidence $(T)$,

degree of indeterminacy between confidence-nonconfidence $(I)$,

and degree of nonconfidence $(F)$.

$Q N$ is a four-dimensional vector that can also be written as: $Q N=(a, b, c, d)$.

$T, I, F$ are herein literal parameters. The multiplication amongst these literal parameters uses the absorbance (prevalence) law, i.e. one parameter absorbs (includes) another (see [9]).

But in specific applications $T, I, F$ may be numerical too (in general, subsets of $[0,1]$ ).

\section{(vi) The Over-/Under-/Off-Indeterminacy}

For OverIndeterminacy we have $I>1$ within the frame of Neutrosophic Overset;

for UnderIndeterminacy we have $I<0$ within the Neutrosophic Underset;

and in general for OffIndeterminacy we have sometimes $I>1$ and other times $I<0$ within the frame of neutrosophic Offset.

For your information, there are cases when the degrees of membership, indeterminacy or nonmembership may be each of them $>1$ or $<0$, and these are happening in our real life applications (see $[10,11]$ ). 


\section{$\underline{\text { Conclusion }}$}

We have presented the broad definition of Indeterminacy, then listed various types of indeterminacies used in neutrosophic set / logic / probability / statistics / measure / precalculus / calculus / algebraic structures, accompanied by applications in our every day life. Indeterminacy is the main distinction between neutrosophic theories and other theories.

\section{References}

[1] F. Smarandache, Neutrosophy. Neutrosophic Probability, Set, and Logic, ProQuest Information \& Learning, Ann Arbor, Michigan, USA, 105 p., 1998; http://fs.unm.edu/eBook-Neutrosophics6.pdf

[2] F. Smarandache, Neutrosophic Quadruple Numbers, Refined Neutrosophic Quadruple Numbers, Absorbance Law, and the Multiplication of Neutrosophic Quadruple Numbers, Ch. 7, pp. 186-193, Chapters in his book: Symbolic Neutrosophic Logic, Europa Nova, Brussels, 194 p., 2015; http://fs.unm.edu/SymbolicNeutrosophicTheory.pdf

[3] F. Smarandache, n-Valued Refined Neutrosophic Logic and Its Applications in Physics, Progress in Physics, 143146, Vol. 4, 2013; http://fs.unm.edu/n-ValuedNeutrosophicLogic-PiP.pdf

[4] F. Smarandache, Introduction to Neutrosophic Measure, Neutrosophic Integral, and Neutrosophic Probability, Sitech \& Educational, Craiova, Columbus, 140 p., 2013; https://arxiv.org/ftp/arxiv/papers/1311/1311.7139.pdf

[5] F. Smarandache: Introduction to Neutrosophic Statistics, Sitech \& Education Publishing, 2014,124 p.; $\underline{\text { http://fs.unm.edu/NeutrosophicStatistics.pdf }}$

[6] J. Kaplan, Neutrosophic Statistics is a generalization of Classical Statistics, Open Library, San Francisco, USA, https://archive.org/details/neutrosophic-statistics?tab=about

https://archive.org/details/neutrosophic-statistics?tab=collection

[7] W. B. Vasantha Kandasamy, F. Smarandache, Fuzzy Cognitive Maps and Neutrosophic Cognitive Maps, Xiquan, Phoenix, 211 p., 2003, http://fs.unm.edu/NCMs.pdf

[8] F. Smarandache, Neutrosophic Precalculus and Neutrosophic Calculus, EdituraNova, Belgium, 2015, http://fs.unm.edu/NeutrosophicPrecalculusCalculus.pdf

[9] F. Smarandache, Neutrosophic Overset, Neutrosophic Underset, and Neutrosophic Offset. Similarly for Neutrosophic Over-/Under-/Off-Logic, Probability, and Statistics, 168 p., Pons Editions, Brussels, Belgium, 2016, http://fs.unm.edu/NeutrosophicOversetUndersetOffset.pdf,

on Cornell University's website: https://arxiv.org/ftp/arxiv/papers/1607/1607.00234.pdf and in France at the HAL international scientific database: https://hal.archives-ouvertes.fr/hal-01340830

[10] N. Martin, Priya. R, F. Smarandache: Decision Making on Teachers' adaptation to Cybergogy in Saturated Interval-valued Refined Neutrosophic overset /underset /offset Environment, International Journal of Neutrosophic Science (IJNS), Volume 12, Issue 2, pp. 58-70, 2020; DOI: 10.5281/zenodo.4268284

[11] Tiago S. dos Reis, Walter Gomide, James A.D.W. Anderson, Construction of Transreal Numbers and Algebraic Transfields, IAENG International Journal of Applied Mathematics, 46:1, IJAM_46_1_03, Advance Online Publication: 15 February 2016. 
[12] F. Smarandache, Matter, Antimatter, and Unmatter, CERN - The European Organization for Nuclear Research, Geneva, Switzerland, 01 Jun 1980, http://cdsweb.cern.ch/record/798551

[13] F. Smarandache, Neutrosophic Set is a Generalization of Intuitionistic Fuzzy Set, Inconsistent Intuitionistic Fuzzy Set (Picture Fuzzy Set, Ternary Fuzzy Set), Pythagorean Fuzzy Set (Atanassov's Intuitionistic Fuzzy Set of second type), q-Rung Orthopair Fuzzy Set, Spherical Fuzzy Set, and n-HyperSpherical Fuzzy Set, while Neutrosophication is a Generalization of Regret Theory, Grey System Theory, and Three-Ways Decision, Journal of New Theory 29 pp. 01-35,2019; also in arXiv, Cornell University, New York City, NY, USA, pp. 1-50, 17-29 November 2019, https://arxiv.org/ftp/arxiv/papers/1911/1911.07333.pdf; and in The University of New Mexico, Albuquerque, USA, Digital Repository, https://digitalrepository.unm.edu/math_fsp/21. 